\title{
The Research on Improved Companding Transformation for Reducing PAPR in Underwater Acoustic OFDM Communication System
}

\author{
Jinqiu Wu, ${ }^{1,2}$ Gang Qiao, ${ }^{1}$ and Xiaofei $\mathbf{Q i}^{1}$ \\ ${ }^{1}$ College of Underwater Acoustic Engineering, Harbin Engineering University, Harbin 150001, China \\ ${ }^{2}$ College of Communication and Electronic Engineering, Qiqihar University, Qiqihar 161000, China \\ Correspondence should be addressed to Jinqiu Wu; jinqiuwu@yahoo.com
}

Received 25 January 2016; Accepted 27 March 2016

Academic Editor: Driss Boutat

Copyright (C) 2016 Jinqiu Wu et al. This is an open access article distributed under the Creative Commons Attribution License, which permits unrestricted use, distribution, and reproduction in any medium, provided the original work is properly cited.

To solve the problem of the high peak-to-average power ratio (PAPR) in Orthogonal Frequency Division Multiplexing (OFDM) for the underwater acoustic communication system, the paper offers a method of reducing PAPR which combines the amplitude limiting and the improved nonlinear transformation. Traditional amplitude limiting technique can reduce PAPR in OFDM system effectively, at the cost of reducing the bit error rate (BER). However the companding transformation has far less computation complexity than SLM or PTS technologies and can improve the BER performance compared to the amplitude limiting technique simultaneously. The paper combines these two kinds of techniques, takes full use of advantages of the two method, and puts forward a low-complexity scheme choosing parameters that are more appropriate to the underwater acoustic field, with the result of improved BER performance even in lower SNR. Both simulation and experiment results show that the new method which combines clipping and companding transformation can effectively reduce the PAPR in the underwater acoustic OFDM communication system and improve the BER performance simultaneously.

\section{Introduction}

Recently, the main research direction of underwater acoustic communication includes high-speed underwater acoustic communication at near distance and low-speed acoustic communication at remote distance [1-3]. High-speed acoustic communication adopts coherent communication technique and multicarrier modulation. OFDM is a kind of multicarrier modulation technique with high spectral efficiency [4-6], and it is widely used in the limited bandwidth underwater acoustic communication. However, OFDM has the defect of high PAPR, which will restrict the linear dynamic range of transmitter's power amplifier and generate clipping distortion which in turn influences the BER performance of the whole system. It will also reduce the accuracy of $A / D$ and D/A convertor and even break the subcarrier's orthogonality in OFDM system [7].

International and domestic research on reducing PAPR mainly divides into following several classes [8-14]: coding technique, probability technique, and signal predistortion technique. The advantage of coding technique is with no signal distortion, while its defect is the high computation cost and the complexity of coding and decoding operation, so coding technique is better for the situation that the subcarriers are less. Probability technique can reduce PAPR effectively, but the computation cost is high and will increase with the growth of the subcarrier number. Signal predisposition technique can reduce PAPR effectively and directly, and the computation cost will not increase with the growth of the number of subcarriers, but it has serious inband interference and out-band noise. Reference [4] provides a method to reduce PAPR with no sideband information, but the computation cost is high. It is necessary to reduce the complexity of the reducing PAPR arithmetic, because of the underwater acoustic channel's characters $[15,16]$. Above all the techniques, signal predisposition technique's advantage is the simpleness of arithmetic, and the computation cost will not increase with the growth of subcarriers [17]; therefore 
it is the most suitable for underwater acoustic OFDM communication system. In signal predisposition technique, clipping is the simplest way to realize and is widely used in previous underwater acoustic OFDM communication. But it is a kind of nonlinear process, which will bring serious in-band interference and out-band noise. At the meantime companding is also with less complexity and can reduce the high PAPR with better BER performance at the same time [18]. The paper is based on this point, according to [7], aiming at the underwater acoustic channel's time-varying and multipath to improve the performance of the underwater acoustic OFDM communication system, choosing parameters that are suitable for the underwater acoustic channel and the parameters can adjust according to the requirements of the application, achieving a compromise between the SNR and $\mathrm{BER}$, and the effectiveness of the arithmetic is proved in the simulation of multipath fading underwater acoustic channel and the water tank experiment.

\section{Definition and Description of the Communication System}

2.1. OFDM Model and Definition of PAPR in Underwater Acoustic Communication. The discrete OFDM signal can be expressed as

$$
s(n)=\frac{1}{\sqrt{N}} \sum_{k=0}^{N-1} d_{k} e^{j(2 \pi n k / N)} .
$$

PAPR can be given by

$$
\operatorname{PAPR}(\mathrm{dB})=10 \log _{10} \frac{\max _{0 \leq n \leq N-1}\left[\left|s_{n}\right|^{2}\right]}{E\left[\left|s_{n}\right|^{2}\right]},
$$

where $s_{n}$ is the data in time-domain after IFFT. The complementary cumulative distribution function (CCDF) of signals that satisfy the Nyquist sampling rate is

$$
\begin{aligned}
P(\operatorname{PAPR}>z) & =1-P(\operatorname{PAPR} \leq z)=1-F(z)^{N} \\
& =1-\left(1-e^{-z}\right)^{N} .
\end{aligned}
$$

$F(z)$ is the cumulative distribution function.

2.2. Companding Transformation Decreases the PAPR of $O F D M$. Companding transformation technique belongs to amplitude limiting technique, whose core idea is to process the signal which has a higher peak power nonlinearly, so that the power will not run out of the dynamic range of the amplifier and avoid the large PAPR [19, 20]. The technique's advantage is simple and its complexity will not increase with the amount of carrier; in addition, it can process inverse transformation at the receiving terminal, which is more suitable for underwater acoustic's complex and limited bandwidth channel.

2.2.1. Traditional Companding Transformation. Companding technique will process the signal nonlinearly before it comes

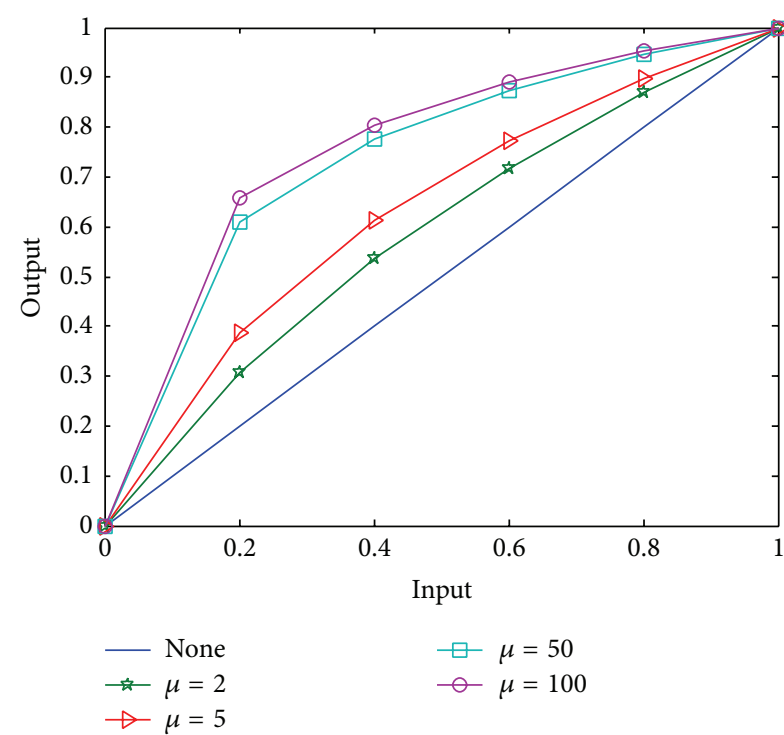

FIgURE 1: Characteristic curve of companding technology.

into the amplifier, amplifying small signal and keeping large signal invariant; therefore the decrease of PAPR is at the cost of increasing the system's average power [21-27].

The traditional companding arithmetic function is as follows:

$$
s_{c n}=C\left(s_{n}\right)=\frac{A \ln \left(1+(\mu / A)\left|s_{n}\right|\right)}{\ln (1+\mu)} \cdot \operatorname{sgn}\left(s_{n}\right),
$$

where $A$ is the peak value of an OFDM signal, $\mu$ is a companding parameter, $s_{n}$ is a discrete OFDM signal, $s_{c n}$ is the OFDM signal after companding, and $\operatorname{sgn}(\cdot)$ is the sign function. The signal received at receiving terminal is demodulated companding signal $r_{n}$ :

$$
\begin{aligned}
s_{c n}^{\prime} & =C^{-1}\left[\left|r_{n}\right|\right] \cdot \operatorname{sgn}\left(r_{n}\right) \\
& =\frac{A^{\prime}}{\mu}\left[\exp \left(\frac{\left|r_{n}\right| \ln (1+\mu)}{A^{\prime}}\right)-1\right] \cdot \operatorname{sgn}\left(r_{n}\right),
\end{aligned}
$$

where $A^{\prime}$ is the peak value of the received signal $r_{n}$.

What is presented in Figure 1 is input/output companding character curve; the figure shows the change of PAPR along with $\mu$. When $\mu$ reaches 50 , there is no decreasing trend of PAPR with increasing of $\mu$, and BER of the system will decrease obviously by increasing of $\mu$; therefore there is no need to choose a large $\mu$.

2.2.2. Improved Companding Arithmetic. The parameters of traditional radio communication companding function utilize the peak value of signal. However, the heavy multipath fading of the underwater acoustic channel, which is influenced by the burst noise coming from marine organisms and ships, leads to the unreliability of the peak value detection at the receiving terminal; therefore the traditional parameter selecting method is not suitable anymore. 


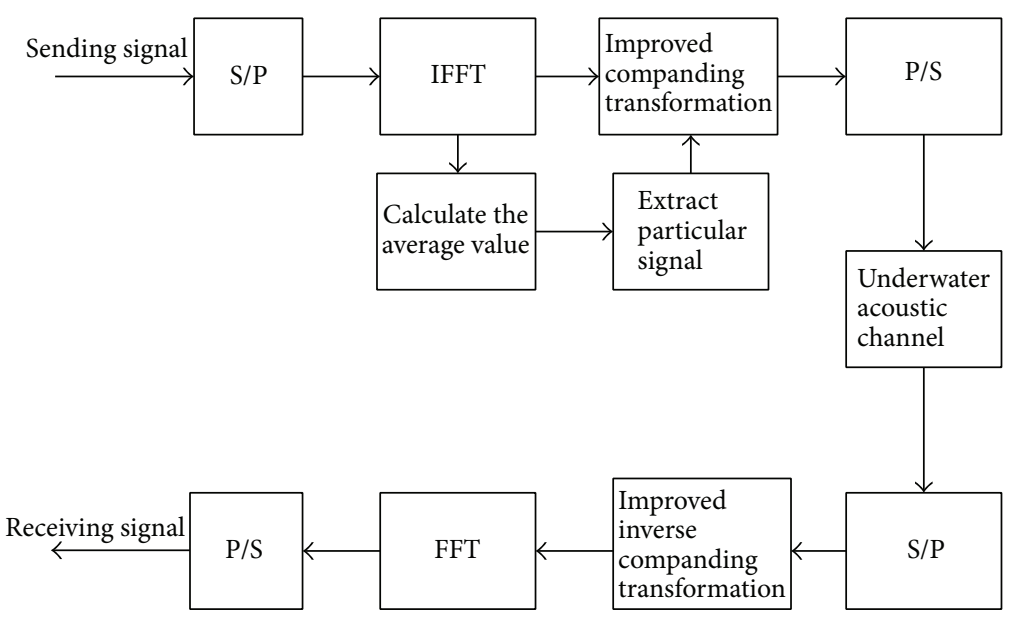

FIGURE 2: Framework of improved companding technology in PAPR reducing for OFDM system.

The improved companding function can be expressed as follows:

$$
s_{c n}= \begin{cases}s_{n}, & \left|s_{n}\right| \subset s_{n 1} \\ \frac{V \ln \left(1+(\mu / V)\left|s_{n}\right|\right)}{\ln (1+\mu)} \cdot \operatorname{sgn}\left(s_{n}\right), & \left|s_{n}\right| \subset s_{n 2},\end{cases}
$$

where $s_{n}$ is the signal before companding, $s_{c n}$ is the signal after companding, signals less than $A$ are expressed as $s_{n 1}, s_{n 2}$ behalves signals which are greater than or equal to $A$, where $A$ is the preset threshold and $V=\operatorname{mean}\left(s_{n 2}\right)$ presents the average value of the signal that exceeds the threshold.

The receiving signal is expressed as $r_{n}, r_{n}$ is composed of $r_{n 1}$ and $r_{n 2}$, and $r_{n 1}=s_{n 1}+w_{n}, r_{n 2}=s_{c n 2}+q_{n}+w_{n}$. $s_{c n 2}$ is the signal of $s_{n 2}$ after companding transformation, $q_{n}$ is quantization noise, and $w_{n}$ is the additive noise that is produced by the channel, and then the inverse transformation in the receiving terminal is:

$$
\begin{aligned}
s_{n}^{\prime} & =C^{-1}\left[\left|r_{n}\right|\right] \cdot \operatorname{sgn}\left(r_{n}\right) \\
& =\left\{\begin{array}{l}
r_{n 1} \\
\frac{V}{\mu} \exp \left(\frac{\left|r_{n 2}\right| \ln (1+\mu)}{V^{\prime}}\right) \cdot \operatorname{sgn}\left(r_{n 2}\right) .
\end{array}\right.
\end{aligned}
$$

Substituting $r_{n 1}$ and $r_{n 2}$ into expression (7),

$$
\begin{aligned}
& s_{n}^{\prime}=C^{-1}\left[\left|r_{n}\right|\right] \cdot \operatorname{sgn}\left(r_{n}\right) \\
& =\left\{\begin{array}{l}
s_{n 1}+w_{n} \\
\frac{V}{\mu} \exp \left(\frac{\left(s_{c n 2}+q_{n}+w_{n}\right) \ln (1+\mu)}{V^{\prime}}\right) \cdot \operatorname{sgn}\left(s_{c n 2}\right) .
\end{array}\right.
\end{aligned}
$$

The improved companding transformation technique block is presented in Figure 2.

2.2.3. BER and the Analysis of Parameters. Researches on the influences of the system's BER performance caused by companding transformation are presented in this segment. Because the arithmetic that this paper adopted is the processing of a part of signal, when using QPSK to modulate signals, the BER of signals which have not been companding satisfies the equation $P_{b}=Q\left[\sqrt{\sigma_{s}^{2} / 2 \sigma_{n}^{2}}\right]$. The influence of the system by companding signal $s_{n 2}$ is discussed in the following.

Companding signal $s_{n 2}$ is expressed as expression (6). Quantization noise variance $\sigma_{q}$ is

$$
\sigma_{q}^{2}=\frac{Q^{2}}{12}=\frac{\left(A / 2^{L-1}\right)^{2}}{12}
$$

where $Q$ is the quantized interval and $L$ is the quantized bit. In the quantification function, the value of $A$ is generally taken as peak value. Signal after inverse companding is

$$
\begin{aligned}
& s_{c n}^{-1} \\
& =\frac{V \exp \left\{\left(\left(s_{c n}+q_{n}+w_{n}\right) / V \operatorname{sgn}\left(s_{c n}\right)\right) \ln (1+\mu)\right\}-V}{\mu \operatorname{sgn}\left(s_{c n}\right)} .
\end{aligned}
$$

Substituting $s_{c n}$ into expression (7):

$$
\begin{aligned}
s_{c n}^{-1} & =\frac{V \exp \left\{\left(\operatorname{sgn}\left(s_{n}\right) \ln \left(1+\mu\left|s_{n} / V\right|\right) \ln (1+\mu) / \ln (1+\mu)\right) / \operatorname{sgn}\left(s_{c n}\right)\right\} \cdot \exp \left\{\left(q_{n}+w_{n}\right) \ln (1+\mu) / V \operatorname{sgn}\left(s_{c n}\right)\right\}-V}{\mu \operatorname{sgn}\left(s_{c n}\right)} \\
& =\frac{\left[V+\mu\left|s_{n}\right|\right] \exp \left\{\left[q_{n}+w_{n}\right] M\right\}-V}{\mu \operatorname{sgn}\left(s_{c n}\right)},
\end{aligned}
$$


where $M=\ln (1+\mu) / V \operatorname{sgn}\left(s_{n}\right)$. According to Taylor series expression exponential function,

$$
\begin{aligned}
e^{\left\{\left[q_{n}+w_{n}\right] M\right\}} \approx 1 & +\left[q_{n}+w_{n}\right] M+\frac{\left\{q_{n}+w_{n}\right\}^{2} M^{2}}{2 !} \\
& +\cdots .
\end{aligned}
$$

In most cases, the quantized noise is tiny; therefore the high order part in the expression can be ignored, and the inverse companding function can be approximated as

$$
s_{c n}^{-1}=s_{n}+\frac{\left[q_{n}+w_{n}\right] V M}{\mu}+s_{n}\left[q_{n}+w_{n}\right] M .
$$

Signals after inverse companding are sent to FFT module at the receiving terminal; the $k$ th subcarrier's source data is $D_{l}(k)$. Consider

$$
\begin{aligned}
& D_{l}(k)=\frac{1}{\sqrt{N}} \sum_{n=0}^{N-1} s_{c}^{-1} e^{-j 2 \pi n k / N}=\frac{1}{\sqrt{N}} \\
& \cdot \sum_{n=0}^{N-1}\left\{s_{n}+\frac{\left[q_{n}+w_{n}\right] V M}{\mu}+s_{n}\left[q_{n}+w_{n}\right] M\right\} \\
& \cdot e^{-j 2 \pi n k / N}=\frac{1}{N} \sum_{n=0}^{N-1}\left\{a_{k} \cos \frac{2 \pi k n}{N}+b_{k} \sin \frac{2 \pi k n}{N}\right\} \\
& \cdot e^{-j 2 \pi n k / N}+\frac{1}{\sqrt{N}} \sum_{n=0}^{N-1} q_{n}\left[\frac{V M}{\mu}+M s_{n}\right] e^{-j 2 \pi n k / N} \\
& +\frac{1}{\sqrt{N}} \sum_{n=0}^{N-1} w_{n}\left[\frac{V M}{\mu}+M s_{n}\right] e^{-j 2 \pi n k / N} .
\end{aligned}
$$

The second item and third item, respectively, express quantizing noise after FFT at receiving terminal and channel noise. The variance of Gaussian channel noise, $\sigma_{k w}$, is

$$
\sigma_{k w}^{2}=\left(\frac{\ln ^{2}(1+\mu)}{\mu^{2}}+\frac{\ln ^{2}(1+\mu)}{V^{2}} E_{s}\right) \sigma_{w}^{2} .
$$

Quantization noise variance $\sigma_{k q}$ is

$$
\begin{aligned}
\sigma_{k q}^{2} & =\frac{1}{N} \sum_{n=0}^{N-1} E\left\{\left[\frac{q_{n} V M}{\mu}+s_{n} q_{n} M\right]^{2}\left[e^{-j 2 \pi k n / N}\right]^{2}\right\} \\
& =E\left\{\frac{q_{n} \ln (1+\mu)}{\mu}\right\}^{2}+E\left\{\frac{s_{n} q_{n} \ln (1+\mu)}{V}\right\}^{2} \\
& \geq \frac{2 \sqrt{E\left(s_{n}^{2}\right)} \sigma_{q}^{2} \ln ^{2}(1+\mu)}{\mu V}=\frac{2 \sigma_{q}^{2} \ln ^{2}(1+\mu)}{\mu^{2}} .
\end{aligned}
$$

If expression is true, then

$$
E\left\{\frac{q_{n} \ln (1+\mu)}{\mu}\right\}^{2}=E\left\{\frac{s_{n} q_{n} \ln (1+\mu)}{V}\right\}^{2} .
$$

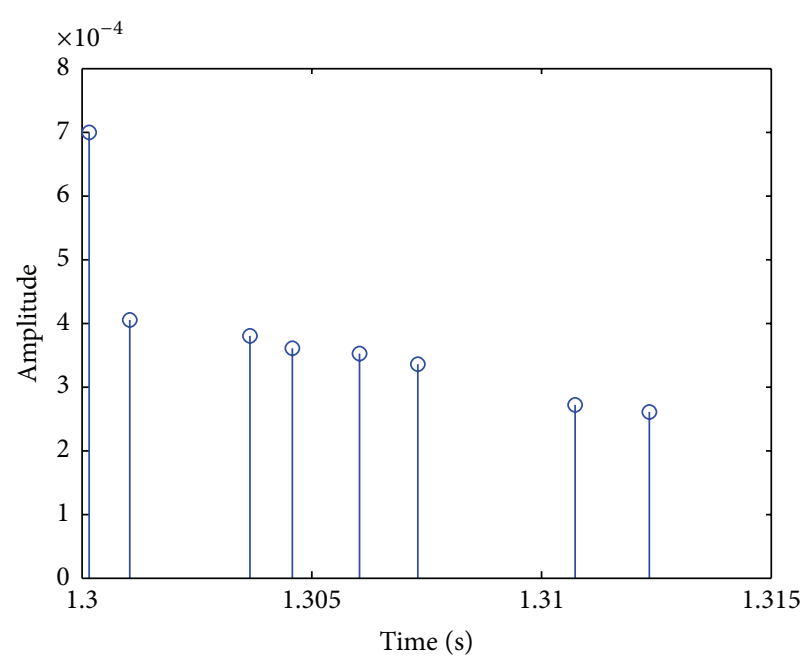

FIGURE 3: Simulation of channel impulse response.

Adopting QPSK modulation, BER satisfies $P_{b}=Q\left[\sqrt{\sigma_{s}^{2} / 2 \sigma_{n}^{2}}\right]$, where $\sigma_{s}$ is the signal variance and $\sigma_{n}$ is the noise variance, and the noise variance consists of quantizing noise and channel noise. Expression (15) and expression (16) show that varying of $\mu$ influences channel noise and quantizing noise. $Q(x)$ is the $Q$ function. In $s_{n 1}, \sigma_{w}^{2}=\sigma_{n}^{2}$; for signals belonging to $s_{n 2}, \sigma_{w}^{2}=\left(\ln ^{2}(1+\mu) / \mu^{2}+\left(\ln ^{2}(1+\mu) / V^{2}\right) E\left(s_{n}^{2}\right)\right) \sigma_{n}^{2}$, the BER of the system is

$$
\begin{aligned}
P_{b} & =Q\left[\sqrt{\frac{\sigma_{s}^{2}}{2 \sigma_{n}^{2}}}\right] \cdot P\left(s_{n}<A\right) \\
& +Q\left[\sqrt{\frac{\sigma_{s}^{2}}{2\left(\ln ^{2}(1+\mu) / \mu^{2}+\left(\ln ^{2}(1+\mu) / V^{2}\right) E\left(s_{n}^{2}\right)\right) \sigma_{n}^{2}}}\right] \\
& \cdot P\left(s_{n} \geq A\right),
\end{aligned}
$$

where $A$ is the average value of the signal.

\section{Simulation and the Water Tank Experiment}

3.1. Simulation Result Analysis. The communication frequent band is $6-12 \mathrm{kHz}$, the length of FFT is 8192 , sample frequent is $48 \mathrm{kHz}$, adopting QPSK modulation, and amount of subcarriers is 1025 . The shallow sea channel is generated by channel simulation software, the depth is 50 meters, the depth of the transducer and hydrophone, respectively, is 22 meters and 10 meters, and the horizontal distance is $2 \mathrm{~km}$.

Figure 3 shows the simulation channel impulse response, and the maximum multipath delay is $12 \mathrm{~ms}$. Figure 4 represents the eigenray of the multipath channel from which the travel path can be observed.

Figure 5 presents the CCDF of these several methods. The improved algorithm can decrease the PAPR effectively. When the CCDF reaches $10^{-1}$ order, the improved arithmetic PAPR has decreased by $2.5 \mathrm{~dB}$ compared to clipping; meanwhile, PAPR has decreased by $1.5 \mathrm{~dB}$ compared to $\mathrm{C}$ transformation. 


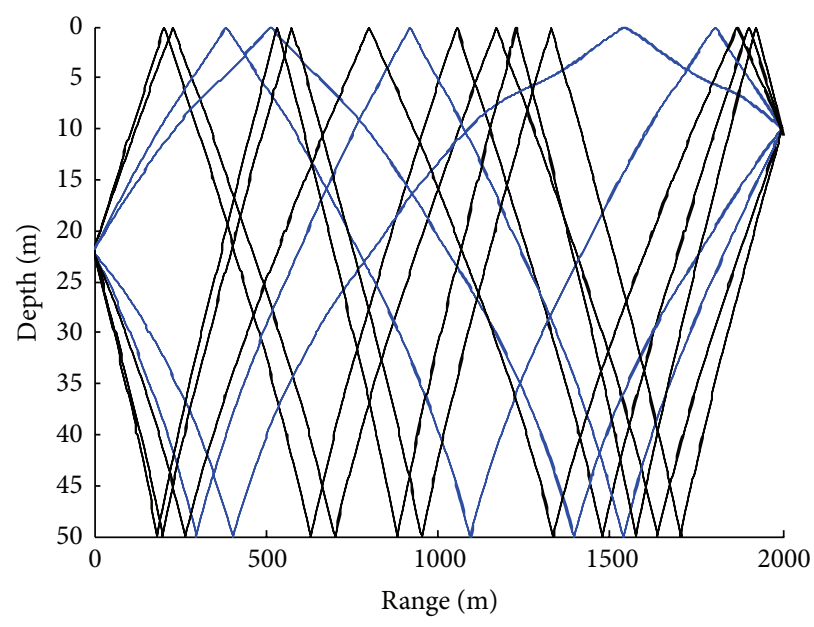

FIgURE 4: Eigenray of the multipath channel.

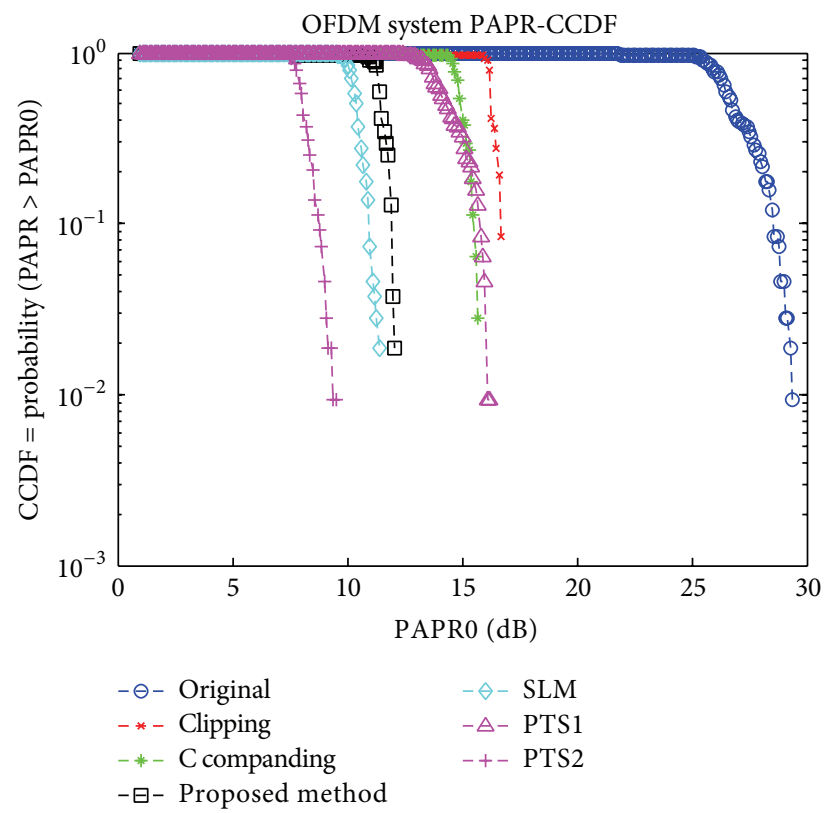

FIgURE 5: Comparison of CCDF.

It also compared the proposed method with Selection Mapping Technique (SLM) and Partial Transmission Sequence (PTS). The two PTS (Partial Transmission Sequence) curves on behalf of two block partition ways are as follows: PTS1 represents the adjacent partition, and interlacing partition style is showed by PTS2. In general the random partition way's capability of reducing PAPR is among the above two division methods; therefore take the above two PTS partition methods, for example. Contrast with SLM and PTS reducing PAPR method, the performance of the proposed method is close to SLM, better than PTS2. But as we know, both SLM and PTS algorithms need to transmit side information, wasting the channel resources, which is precious in underwater acoustic communication. The different reducing PAPR performance in two PTS methods, mainly because of the sources, which transmitted in the system, is with high PAPR and has not been fully interleaved.

Figure 6 represents absolute amplitude of OFDM signal before and after processing, with the condition that transformed signal's average power is invariable; this paper compares several methods' capacities of decreasing the PAPR. Figures 6(b), 6(c), 6(d), and 6(e) represent the clipping, C transformation, SLM, and improved method.

The influence of different decreasing PAPR method on system BER performance is presented in Figure 7. Clipping has the largest interference. When SNR is $20 \mathrm{~dB}$, the BER of the improved method is $10^{-1}$ lower than companding transformation. When SNR is lower than $12 \mathrm{~dB}$, the proposed algorithm's BER performance is nearly equivalent to SLM. As simulation result shows, the improved arithmetic can 


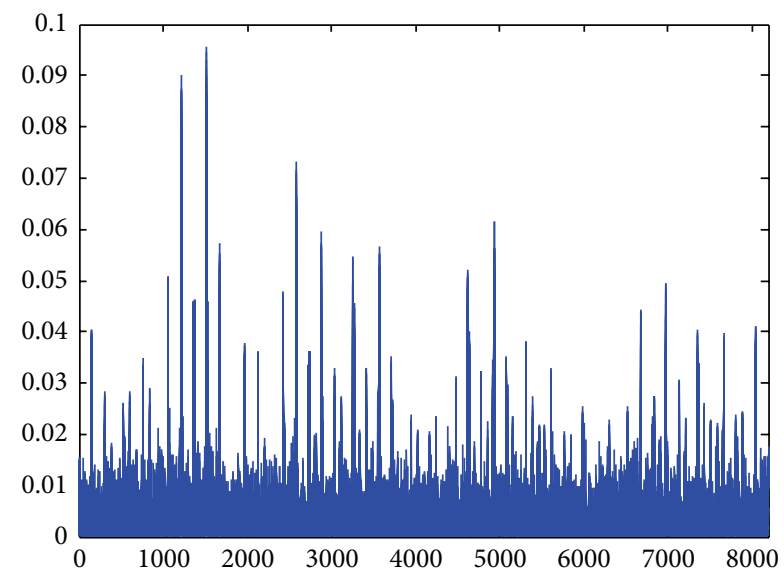

(a) Original

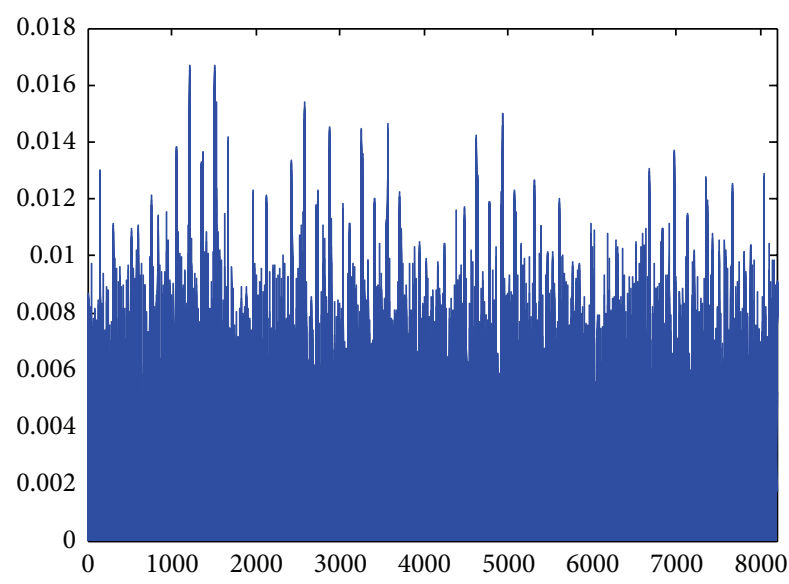

(c) C transformation

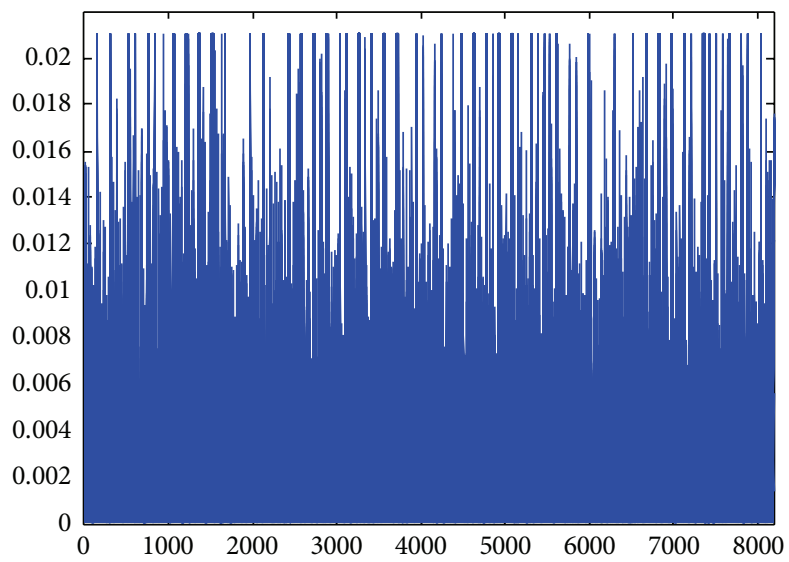

(b) Clipping

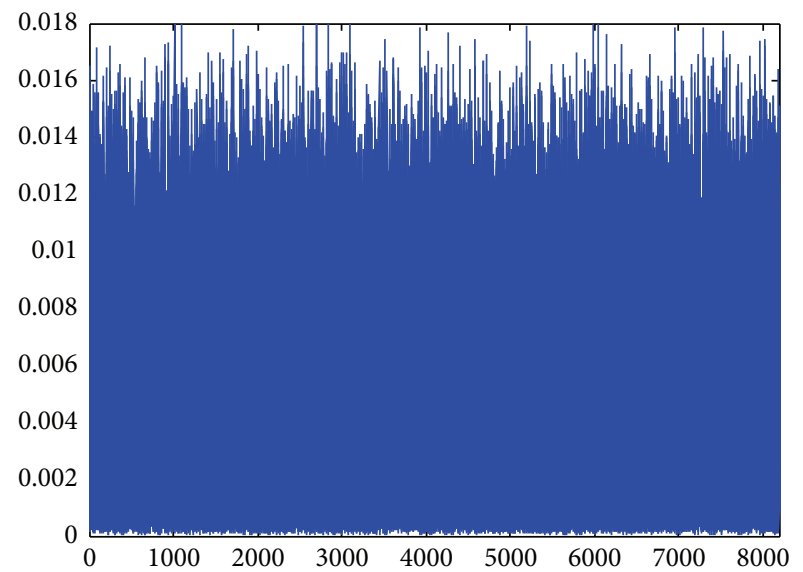

(d) SLM

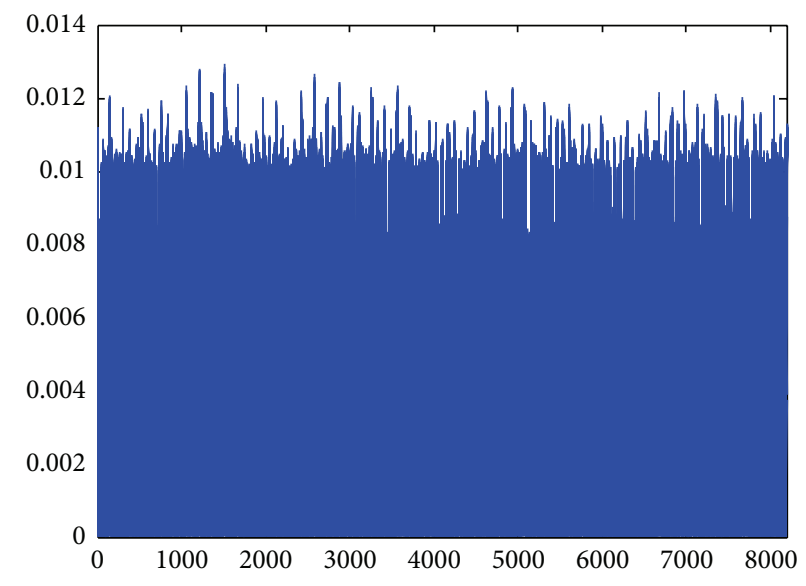

(e) Proposed method

FIGURE 6: Amplitude of OFDM signals before and after processing.

improve the performance of the system and decrease PAPR simultaneously. It can also improve the system's transmission efficiency compared to SLM and PTS and with less computational complexity.

3.2. Water Tank Experiment Result Analysis. In 2015, at Harbin Engineering University, the experiment was done in the channel water tank. There are sands at the bottom of the water tank, valid depth is about 4 meters, the length is 45 meters, and width is 6 meters, with the silence wedge around. Transducer is at 1 meter underneath the surface, hydrophone is at 1.5 meters underneath the surface, and the horizontal distance is about 14 meters.

At first, we produce a transmission signal using MATLAB software and translate it into a WAV file, transmitting the signal from computer's sound card. The signal goes through 


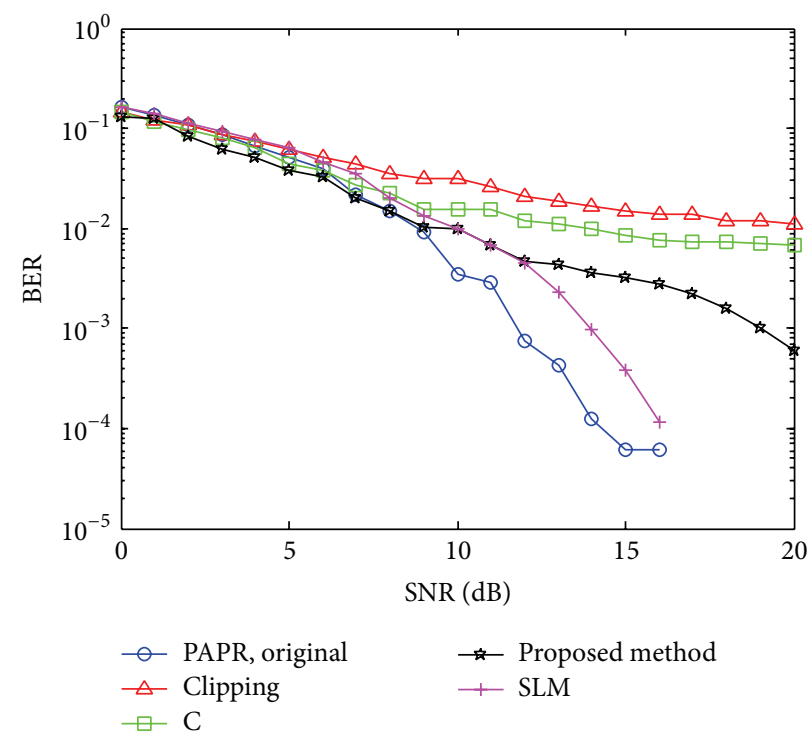

FIgURE 7: Influence of different method on OFDM system.

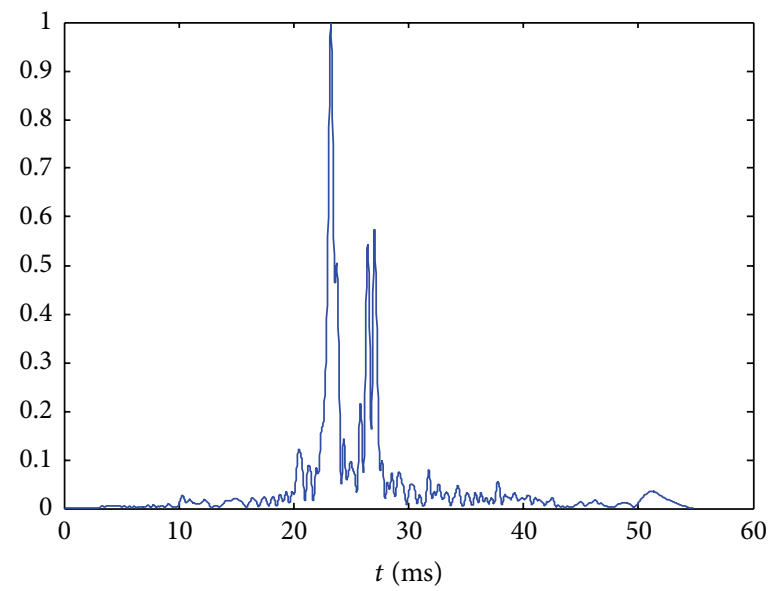

FiguRE 8: Impulses response of tank channel.

the power amplifier, transmitted by a transducer, passing the underwater acoustic channel, received by the hydrophone. The signal is collected and stored by a computer for distant processing.

Figure 8 is the channel impulses response of the water tank that reflects the experimental channel environment.

Figure 9 represents the demodulation result of a different method to decrease PAPR in OFDM experiment. The average statistics BER of clipping arithmetic, C transformation, SLM algorithm, and the improved arithmetic are, respectively, $8.8 \times 10^{-3}, 7.5 \times 10^{-3}, 9.4 \times 10^{-4}$, and $8.9 \times 10^{-4}$. The result shows that the improved arithmetic can decrease PAPR with BER decreasing. The experiment proved that the improved arithmetic is in accordance with the simulation result.

\section{Conclusion}

At present, the technique to decrease PAPR is at the cost of increasing power, increasing BER, decreasing the data rate, and adding computational complexity. In practical, we need to choose a suitable method according to each influence factor of an OFDM system. In an underwater acoustic OFDM system, the transmitting of data is in severe surroundings. The band-width is limited in the acoustic channel, so it enlarges the influence of delay spread and frequency selective fading compared to wireless channels. This requires the method which decreases the PAPR to be of low complexity and to keep the signal recovered exactly with less influence in the meantime. The paper takes advantage of amplitude limiting arithmetic and $\mathrm{C}$ transformation, combining them to apply underwater acoustic OFDM communication system. After the simulation comparison of amplitude limiting arithmetic, $\mathrm{C}$ transformation, and improved arithmetic, we get the result that the improved arithmetic can both decrease PAPR and improve the performance of the system, with the advantage of low computation complexity and being easy to realize. The computation complexity will not be influenced by the amount 


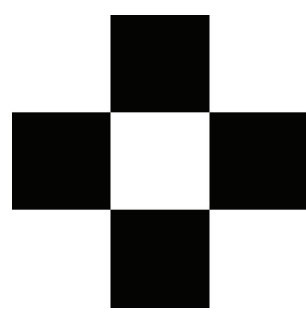

(a)

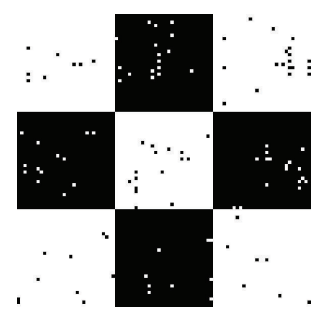

(b)

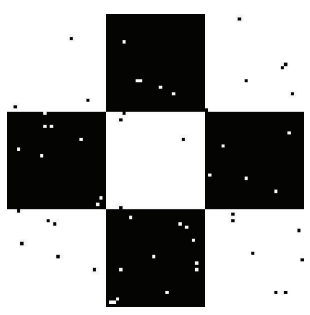

(c)

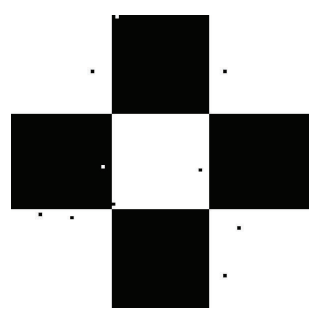

(d)

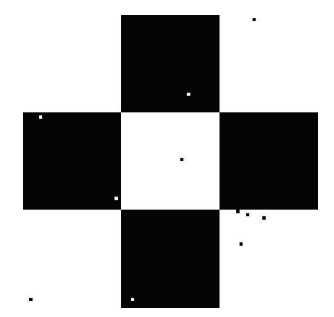

(e)

Figure 9: Send and receive figure: (a) original, (b) clipping, (c) C companding, (d) SLM, and (e) the improved method.

of subcarriers, so it is suitable to apply in underwater acoustic communication system with a limited band-width.

\section{Competing Interests}

The authors declare that they have no competing interests.

\section{Acknowledgments}

The authors thank the project of the National Natural Science Foundation of China no. 61431004, no. 6140114, and no. 11274079 and the Teaching and Research Project of Qiqihar University no. 2014087.

\section{References}

[1] P. Kumar and P. Kumar, "A comparative study of spread OFDM with transmit diversity for underwater acoustic communications," Wireless Personal Communications, vol. 83, no. 1, pp. 6986, 2015.

[2] J.-W. Yin, S. Yang, P.-Y. Du, Y. Yu, and Y. Chen, "Code divided multiple access underwater acoustic communication based on active acoustic intensity average," Acta Physica Sinica, vol. 61, no. 6, pp. 329-335, 2012 (Chinese).

[3] Y.-L. Yin, F. Zhou, G. Qiao, and S.-Z. Liu, "Orthogonal multicarrier $M$-ary cycle shift keying spread spectrum underwater acoustic communication," Acta Physica Sinica, vol. 62, no. 22, pp. 254-263, 2013 (Chinese).

[4] W. Wang, G. Qiao, and S.-Y. Xing, "A selective mapping peakto-average power ratio reduction algorithm without side information for underwater acoustic multiple-input multiple-output orthogonal frequency division multiplexing communication," Acta Physica Sinica, vol. 62, no. 18, Article ID 184301, 2013 (Chinese).

[5] T. Kang and R. A. Iltis, "Iterative carrier frequency offset and channel estimation for underwater acoustic OFDM systems," IEEE Journal on Selected Areas in Communications, vol. 26, no. 9, pp. 1650-1661, 2008.

[6] C. Polprasert, J. A. Ritcey, and M. Stojanovic, "Capacity of OFDM systems over fading underwater acoustic channels," IEEE Journal of Oceanic Engineering, vol. 36, no. 4, pp. 514-524, 2011.

[7] T. Jiang and G. Zhu, "Nonlinear companding transform for reducing peak-to-average power ratio of OFDM signals," IEEE Transactions on Broadcasting, vol. 50, no. 3, pp. 342-346, 2004.

[8] D.-W. Lim, S.-J. Heo, and J.-S. No, "An overview of peak-toaverage power ratio reduction schemes for OFDM signals,"
Journal of Communications and Networks, vol. 11, no. 3, pp. 229239, 2009.

[9] G. E. Arrobo and R. D. Gitlin, "Improving the performance of OFDM-based vehicular systems through diversity coding," Journal of Communications and Networks, vol. 15, no. 2, pp. 132$141,2013$.

[10] G. Wunder, R. F. H. Fischer, H. Boche, S. Litsyn, and J.-S. No, "The PAPR problem in OFDM transmission: new directions for a long-lasting problem," IEEE Signal Processing Magazine, vol. 30, no. 6, pp. 130-144, 2013.

[11] H.-B. Jeon, K.-H. Kim, J.-S. No, and D.-J. Shin, "Bit-based SLM schemes for PAPR reduction in QAM modulated OFDM signals," IEEE Transactions on Broadcasting, vol. 55, no. 3, pp. 679-685, 2009.

[12] S.-J. Heo, H.-S. Noh, J.-S. No, and D.-J. Shin, "A modified SLM scheme with low complexity for PAPR reduction of OFDM systems," IEEE Transactions on Broadcasting, vol. 53, no. 4, pp. 804-808, 2007.

[13] S.-H. Wang, J.-C. Sie, C.-P. Li, and Y.-F. Chen, "A lowcomplexity PAPR reduction scheme for OFDMA uplink systems," IEEE Transactions on Wireless Communications, vol. 10, no. 4, pp. 1242-1251, 2011.

[14] P. Banelli and S. Cacopardi, "Theoretical analysis and performance of OFDM signals in nonlinear AWGN channels," IEEE Transactions on Communications, vol. 48, no. 3, pp. 430-441, 2000.

[15] C. R. Berger, S. L. Zhou, J. C. Preisig, and P. Willett, "Sparse channel estimation for multicarrier underwater acoustic communication: from subspace methods to compressed sensing," IEEE Transactions on Signal Processing, vol. 58, no. 3, pp. 17081721, 2010.

[16] M. Stojanovic and J. Preisig, "Underwater acoustic communication channels: propagation models and statistical characterization," IEEE Communications Magazine, vol. 47, no. 1, pp. 84-89, 2009.

[17] K. Bandara, P. Niroopan, and Y. Chung, "PAPR reduced OFDM visible light communication using exponential nonlinear companding," in Proceedings of the IEEE International Conference on Microwaves, Communications, Antennas and Electronics Systems (IEEE COMCAS '13), pp. 1-5, Tel Aviv, Israel, October 2013.

[18] E. Singh, M. Arif, V. Shrivastava, and R. Bhatia, "Nonlinear companding technique for PAPR reduction in OFDM," in Proceedings of the 1st International Conference on Signal Propagation and Computer Technology (ICSPCT '14), pp. 801-805, Ajmer, India, July 2014.

[19] N. S. L. P. Kumar, A. Banerjee, and P. Sircar, "Modified exponential companding for PAPR reduction of OFDM signals," in Proceedings of the IEEE Wireless Communications and Networking Conference, pp. 1345-1350, Hong Kong, 2007. 
[20] M. J. Omidi, A. Minasian, H. Saeedi-Sourck, K. Kasiri, and I. Hosseini, "PAPR reduction in OFDM systems: polynomialbased compressing and iterative expanding," Wireless Personal Communications, vol. 75, no. 1, pp. 103-118, 2014.

[21] M. Hu, Y. Li, Y. Liu, and H. Zhang, "Parameter-adjustable piecewise exponential companding scheme for peak-to-average power ratio reduction in orthogonal frequency division multiplexing systems," IET Communications, vol. 8, no. 4, pp. 530536, 2014.

[22] S. Peng, S. Yuehong, Z. G. Yuan, and W. Jian, "PAPR reduction of LOFDM signals with an efficient nonlinear companding transform," in Proceedings of the International Conference on Wireless Communications and Signal Processing (WCSP '13), pp. 1-6, Hangzhou, China, October 2013.

[23] H. Ochiai and H. Imai, "Performance analysis of deliberately clipped OFDM signals," IEEE Transactions on Communications, vol. 50, no. 1, pp. 89-101, 2002.

[24] L. Wang and C. Tellambura, "Analysis of clipping noise and tone-reservation algorithms for peak reduction in OFDM systems," IEEE Transactions on Vehicular Technology, vol. 57, no. 3, pp. 1675-1694, 2008.

[25] U.-K. Kwon, D. Kim, and G.-H. Im, "Amplitude clipping and iterative reconstruction of MIMO-OFDM signals with optimum equalization," IEEE Transactions on Wireless Communications, vol. 8, no. 1, pp. 268-277, 2009.

[26] R. J. Baxley, C. Zhao, and G. T. Zhou, "Constrained clipping for crest factor reduction in OFDM," IEEE Transactions on Broadcasting, vol. 52, no. 4, pp. 570-575, 2006.

[27] Y. Wang, W. Chen, and C. Tellambura, "Genetic algorithm based nearly optimal peak reduction tone set selection for adaptive amplitude clipping PAPR reduction," IEEE Transactions on Broadcasting, vol. 58, no. 3, pp. 462-471, 2012. 


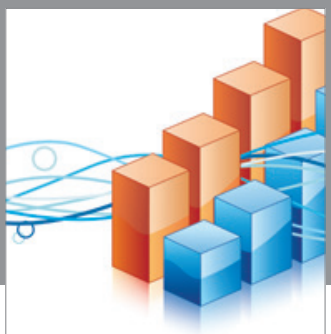

Advances in

Operations Research

vatem alat4

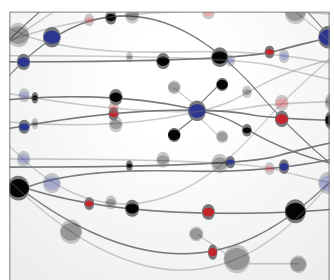

\section{The Scientific} World Journal
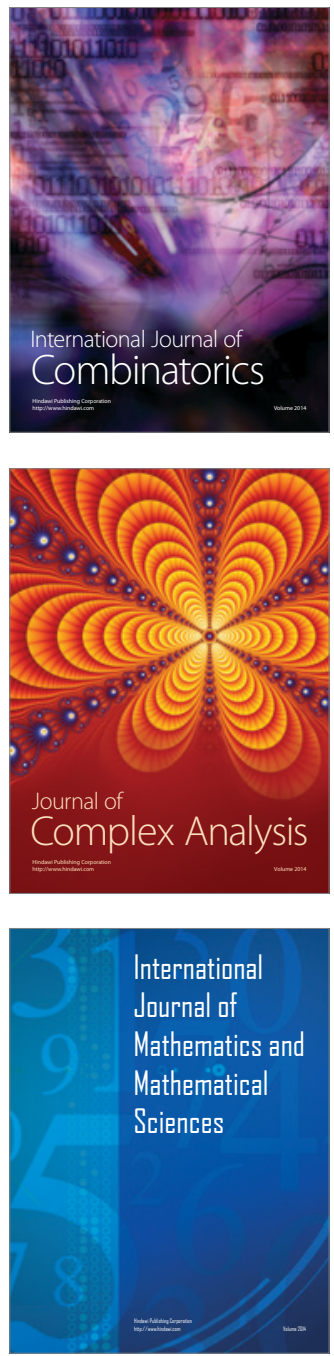
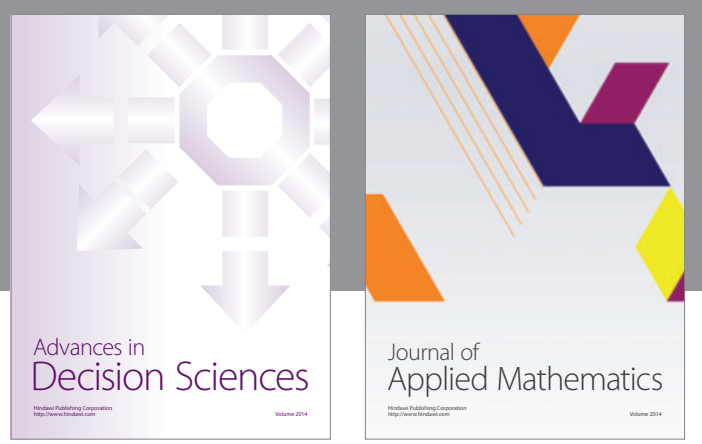

Algebra

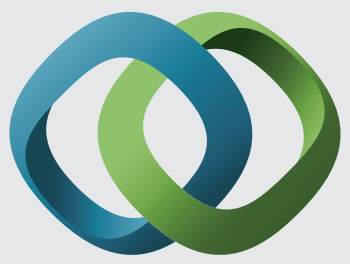

\section{Hindawi}

Submit your manuscripts at

http://www.hindawi.com
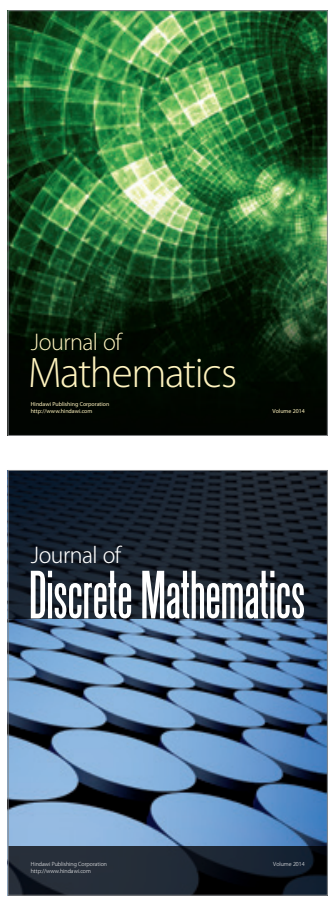

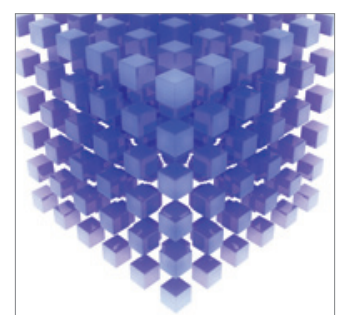

Mathematical Problems in Engineering
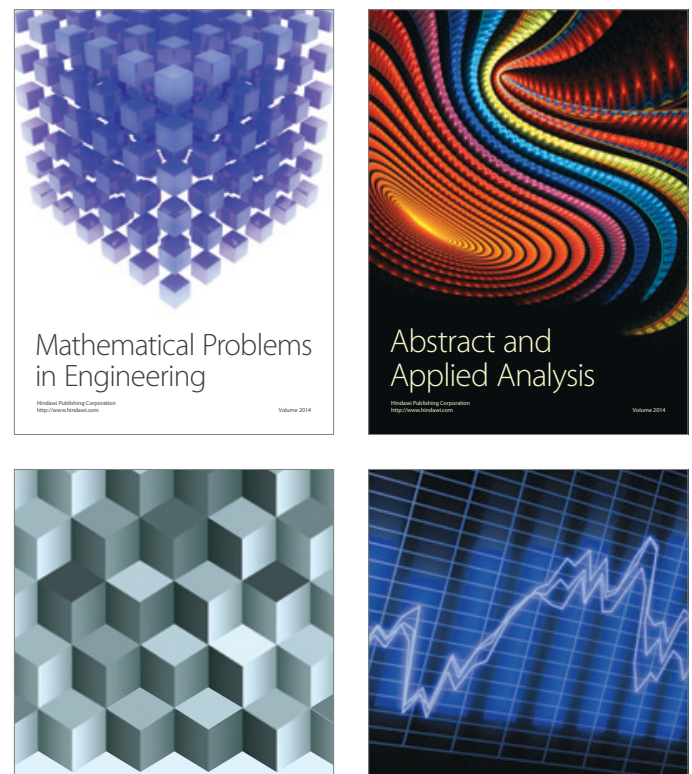

Journal of

Function Spaces

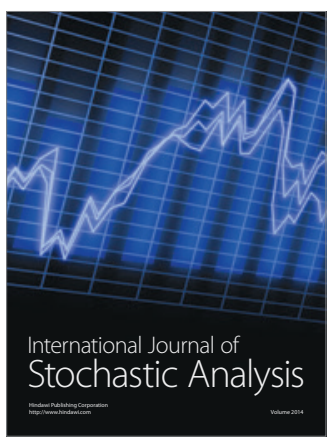

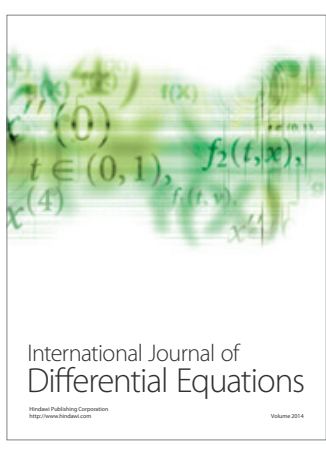
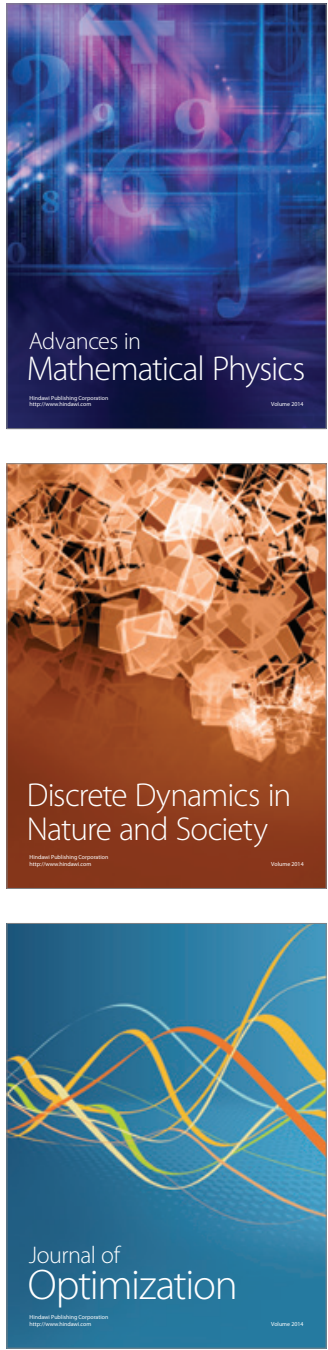